\title{
Los cambios de mirada necesarios sobre las figuras
}

Artículo recibido: 29-07-2010 | Artículo aprobado: 13-08-2010

Necessary changes in the way we see figures

\section{Raymond Duval*}

Titulo original: Les changements de regard nécessaire sur les figures

Publicado en la revista Grand No.76, pp. 7-27, 2005

Traducción: Martín Eduardo Acosta Gempeler**

\begin{abstract}
Resumen: El trabajo teórico en geometría requiere una transformación de la manera de ver y descomponer las figuras. Es necesario un pasar de una descomposición en formas 2D a una descomposición en redes de rectas. En este artículo estudiamos las dificultades de este cambio, y proponemos una selección cuidadosa de tareas de reproducción de dibujos con instrumentos no estándar: los moldes y las plantillas, que pueden favorecerlo.
\end{abstract}

Palabras clave: deconstrucción dimensional, percepción, descomposición de figuras, ensamblaje por yuxtaposición, ensamblaje por superposición, reconocimiento de propiedades geométricas.

\begin{abstract}
The theoretical work in geometry requires a transformation of the way people see and separate figures in basic elements. One has to change from 2D shapes decomposition to a decomposition in networks of straight lines. In this paper we study the difficulties of this change, and propose a careful selection of reproduction tasks using no standard instruments: the molds and the insoles, which can favor it.
\end{abstract}

Key-words: Dimensional deconstruction, perception, decomposition of figures, assembly by juxtaposition, overlap assembly, geometric properties recognition.

* Marc Godin. IUFM Nord - Pas de Calais

* Escuela de Matemáticas, Universidad Industrial de Santander. martin@matematicas.uis.edu.co 
Los cambios de mirada necesarios sobre las figuras Raymond Duval | Traducción: Martín Eduardo Acosta Gempeler

\section{Introducción}

La utilización de figuras (su análisis e interpretación) es uno de los puntos clave para introducir a los alumnos en el mundo de la geometría, pero también es lugar de profundos equívocos didácticos.

En efecto, la organización de los objetivos de enseñanza, desde la primaria, da prioridad a las rectas, a sus relaciones, a sus propiedades. El trabajo sobre figuras de base (triángulo, cuadrado...) se hace en función de las rectas. Esto conduce a valorar las figuras "un D" (1D)1 o las configuraciones de figuras 1D (rectas paralelas, rectas perpendiculares) más que las figuras 2D, o al menos a considerarlas de igual valor.

Ese orden de introducción de los conocimientos choca con la manera como se interpretan y se perciben las figuras fuera de las matemáticas. Aquello que se reconoce como una forma 2D, no se descompone perceptivamente en una red de formas 1D. En otras palabras, existe una prioridad cognitiva de las figuras 2D sobre las figuras 1D. Y ni hablar de los puntos, que sólo se ven si son vértices de polígonos, o se marcan de manera explícita. En otras palabras, la deconstrucción dimensional de las formas que requiere la introducción de los

1 Asumimos aquí las anotaciones clásicas 1D, 2D, $3 D$ en las que $D$ es una abreviación de la palabra dimensión, abreviación que también se utiliza para las imágenes -se dice por ejemplo "imagen en 3D". No es superfluo recordar que las primeras definiciones que da Euclides de una línea o de una superficie son una descripción de la deconstrucción dimensional de las figuras: "los límites de una superficie son líneas" (definición seis). "una figura es lo que está contenido por algunos límites" (definición 14). Euclides coloca la deconstrucción dimensional de las figuras al comienzo de la geometría, como el umbral que hay que atravesar para entrar en la construcción o el descubrimiento de conocimientos geométricos. Esto le parece tan evidente que no se detiene allí. ¿Sucede lo mismo con los alumnos jóvenes? conocimientos geométricos va en contra de los procesos espontáneos de identificación visual de las formas.

Este conflicto entre la práctica geométrica de las figuras y el modo cognitivo de su reconocimiento implica un problema difícil y decisivo para la enseñanza de la geometría: ¿Cómo hacer para que los alumnos cambien la mirada sobre las figuras? ¿Cómo hacerlos pasar de una mirada centrada en las superficies y sus contornos a una mirada que hace aparecer la red de rectas y de puntos subyacente a las diferentes figuras estudiadas en la escuela? Este paso exige un desarrollo de las capacidades de análisis visual de las figuras. Sin esta transformación de la manera espontánea y predominante de ver, todas las formulaciones de propiedades geométricas corren el riesgo de ser formulaciones vacías.

Podremos causar sorpresa -e incluso indignación- afirmando no solamente que tal paso exige un trabajo de varios años, sino sobre todo que los tipos de actividades que se proponen habitualmente en relación con las figuras no permiten para nada que los alumnos cambien su manera de ver. Los tipos de actividad más corrientes conciernen a la reproducción o la construcción de figuras en 1D. Pero en esas actividades no se le presta suficiente atención a las restricciones específicas de análisis visual que cada tipo de instrumento impone. Por ejemplo, la reproducción no constituye un sólo tipo de tarea, sino que hay tantos tipos de tareas de reproducción como tipos de instrumentos utilizados. La variación de los instrumentos es una variable didáctica esencial, que los estudios consagrados al análisis de las figuras no toman en cuenta (Bouleau 2001).

El problema que vamos a estudiar en este artículo es el de la relación con las figuras en la enseñanza de la geometría y el 
de los medios para hacerla evolucionar. Lo haremos abordando tres preguntas:

- ¿Cómo analizar una figura para ser capaz de ver lo que hay que ver geométricamente?

- ¿Qué tipos de tarea y qué figuras para esas tareas son necesarias para hacer cambiar la manera de ver de los alumnos?

- ¿Cómo organizar actividades centradas en el análisis de las figuras?

\section{Ver una figura y analizarla}

\section{Tres caminos diferentes para analizar una figura}

El análisis de una figura puede hacerse desde tres caminos diferentes. El primero es evidentemente el de la percepción: el análisis se hace en función de las formas (o unidades figurales) que se reconocen y de las propiedades visuales de esas formas.

Los otros dos son los que la enseñanza de la geometría trata de desarrollar. Por una parte, está el conocimiento de las propiedades geométricas que deben movilizarse en función de hipótesis dadas: para analizar una figura, deben utilizarse las propiedades geométricas y no las formas visualmente reconocidas. Por otra parte, están los diferentes instrumentos que pueden utilizarse para reproducir o para construir una figura: el análisis de la figura depende de los procedimientos de reproducción o de construcción que el instrumento impone.

Vamos a presentar brevemente cada uno de esos tres caminos, insistiendo particularmente en el análisis instrumental. En efecto, al jugar con la variable que ofrecen los instrumentos en una situación de reproducción, se invertirá en los alumnos la predominancia fuerte y durable de un análisis perceptivo con respecto a un análisis geométrico de figuras. Por ejemplo, hay instrumentos que hacen analizar una figura en términos de unidades $2 \mathrm{D}$ y otros en términos de unidades 1D. Las tareas de reproducción serán radicalmente diferentes según el tipo de instrumentos escogidos.

En función de las formas (o unidades figurales) que se reconocen y de las propiedades visuales de esas formas

Las formas 2D corresponden a contornos cerrados. Podemos entonces distinguir en una figura tantas formas 2D como contornos cerrados haya. Pero en realidad, las cosas no son tan simples, pues en una figura pueden verse ...

... tantas formas como contornos cerrados hay. En este caso se hablará de "ensamblaje por yuxtaposición".

... menos formas que contornos cerrados hay. En este caso se hablará de "ensamblaje por superposición".

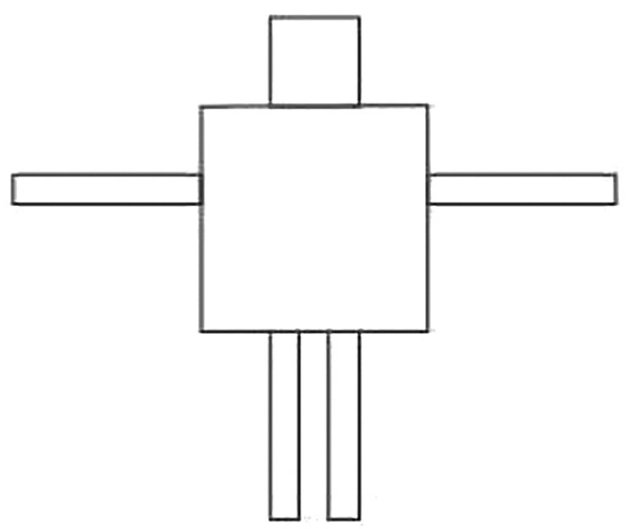

Ensamblaje por yuxtaposición 
Los cambios de mirada necesarios sobre las figuras Raymond Duval | Traducción: Martín Eduardo Acosta Gempeler

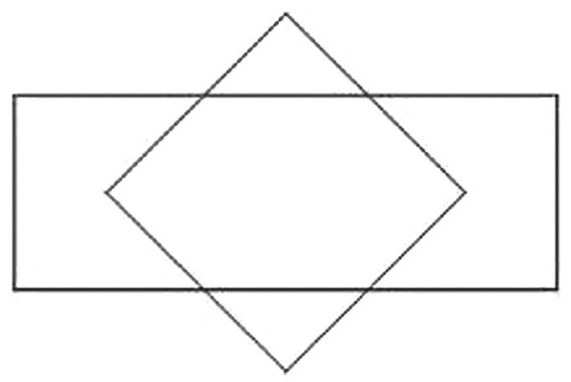

Ensamblaje por superposición

Figura 1: Dos tipos de ensamblaje figural de formas $2 \mathrm{D}$.

Vemos entonces la diferencia entre un ensamblaje por yuxtaposición y de un ensamblaje por superposición. En un ensamblaje por superposición, hay una reducción importante de las formas efectivamente reconocidas. El primer interés didáctico de esta distinción para la elección de las figuras sobre las que se hará trabajar a los alumnos es el siguiente: los ensamblajes por superposición privilegian visualmente la prolongación de los trazados reconocidos como pertenecientes a una forma y no a otra. Como lo veremos más adelante, la actividad de prolongación de trazados juega un papel esencial en el paso de las superficies a las líneas. En el ejemplo anterior, las dos formas superpuestas fueron trazadas independientemente una de la otra, lo que impide saber si el cuadrado está encima o debajo del rectángulo; corresponde a la superposición de dos formas transparentes. Pero después de hacer la elección, pueden borrarse algunos trazos.

Esta distinción necesita precisiones importantes. Por ejemplo, nada impide ver la figura de la izquierda como un ensamblaje por superposición: los dos "brazos" como una sola y misma forma rectangular y el contorno de la "cabeza" como prolongación de las "piernas". En este ejemplo, es sólo el carácter "figurativo" de la figura, el que constituye el obstáculo para ese cambio de mirada: parece en su totalidad la silueta de un muñeco y cada parte de la figura, yuxtapuesta a las otras partes, se parece a una parte del cuerpo. Esta figura es fuertemente icónica en el sentido de Pierce. Entonces sólo una actividad gráfica de prolongación de trazados dará a esta figura su carácter geométrico y borrará su fuerte iconicidad o su figuratividad.

Por el contrario, hay una resistencia perceptiva intrínseca a ver la figura de la derecha (figura 1) como un ensamblaje por yuxtaposición, por ejemplo como un ensamblaje de tres formas de tipos diferentes: triángulos, pentágonos cóncavos y hexágonos convexos.

De hecho, para pasar de un ensamblaje por superposición a un ensamblaje por yuxtaposición como en la siguiente figura de la derecha (figura 2), en general es insuficiente una actividad gráfica. Por eso es indispensable el recurso al coloreado, o a piezas materiales de rompecabezas que se pueden desplazar y ajustar unas a las otras. En otras palabras, hay que abandonar la actividad gráfica y cambiarla por manipulaciones físicas de moldes o por color.

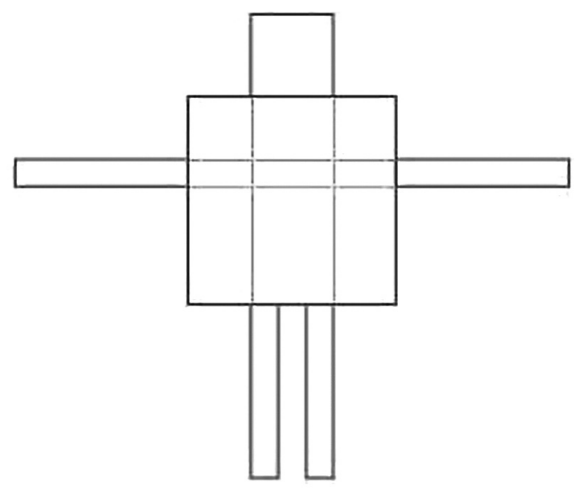

La prolongación de los trazos invierte un ensamblaje por yuxtaposición en un ensamblaje por superposición 


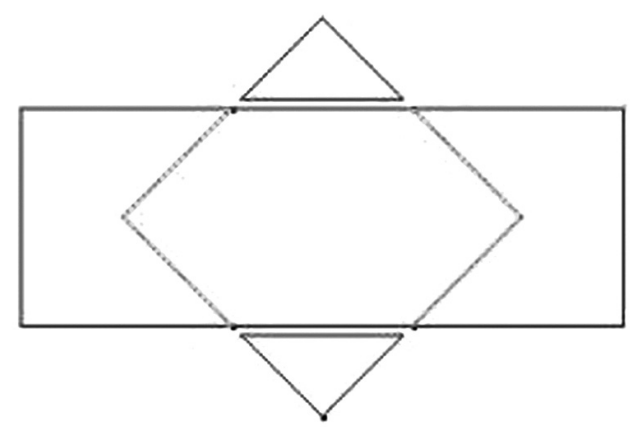

La descomposición en tantas formas como contornos cerrados transforma un ensamblaje por superposición en un ensamblaje por yuxtaposición

Figura 2: Cambio de mirada e inversión del tipo de ensamblaje.

Vemos entonces la ambivalencia gestaltista de toda figura plana cuando se distinguen por lo menos dos contornos cerrados (dos unidades figurales 2D). Esta ambivalencia se debe al hecho de que puede ser vista como un ensamblaje por yuxtaposición o como un ensamblaje por superposición. Pero cuando una de esas dos miradas se impone-lo que a menudo sucede a primera vista- realizar el cambio requiere una actividad gráfica de prolongación de trazos o una actividad de manipulación y desplazamiento de moldes.

Sea como sea, en ambos casos, la mirada sólo identifica las formas 2D, las superficies, que corresponden a los contornos cerrados, y no las formas 1D que son los bordes o separaciones, puntos extremos de las formas 2D.

Esto condiciona el primer trabajo de la enseñanza: hacer pasar de un análisis visual de las figuras en términos de ensamblajes de superficies (formas 2D) a un análisis visual en términos de ensamblajes de líneas (formas 1D).
En función de las hipótesis dadas y del conocimiento que se tiene de las propiedades geométricas

Lo que se entiende generalmente por "figura" es la superposición de marcas discursivas (hipótesis, codificaciones) -que darán propiedades geométricas- a la representación de una forma simple 2D o de un ensamblaje de formas 2D. Naturalmente, se supone que las propiedades geométricas van a dirigir el análisis de la representación visual, que siempre es particular y modificable. En otras palabras, se espera que la representación visual se subordine cognitivamente a las informaciones geométricas que se les superponen.

Generalmente, cuando se habla de "figura", se piensa que no se puede hacer nada con una forma visual como la de abajo

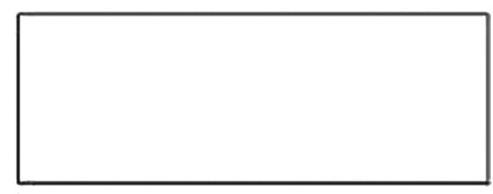

pero

Que son necesarias por lo menos informaciones como, por ejemplo las que están aquí abajo

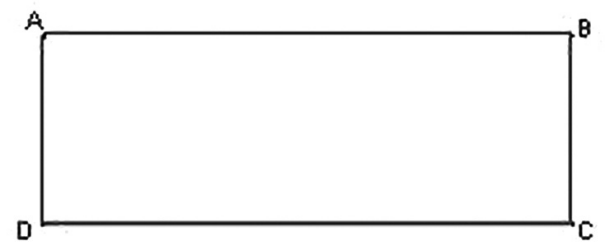


Los cambios de mirada necesarios sobre las figuras Raymond Duval | Traducción: Martín Eduardo Acosta Gempeler

0

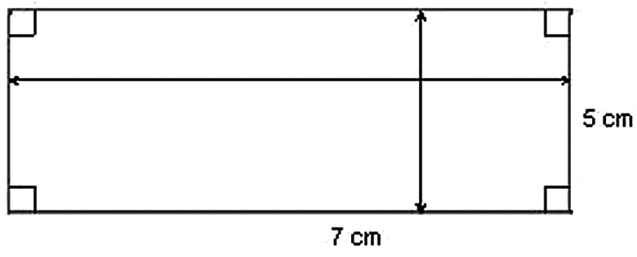

Figura 3. Las dos caras de una figura en geometría.

Es esta subordinación cognitiva de la forma visual a las informaciones dadas o codificadas la que constituye un verdadero obstáculo para entrar en la geometría. En efecto, para que tal superposición funcione y funcione como un todo en el que las propiedades geométricas prevalecen sobre las evidencias visuales, es necesaria una condición cognitiva. Hay que ser capaz de reorganizar la percepción de las formas $2 \mathrm{D}$, es decir una percepción centrada en los contornos cerrados, en la percepción de un conjunto de unidades visuales 1D, pues las propiedades geométricas se refieren esencialmente a relaciones entre esas unidades 1D. Eso quiere decir que analizar una figura en función del conocimiento que se tiene de las propiedades geométricas supone la deconstrucción dimensional de las representaciones visuales que se quieren articular con las propiedades geométricas. Lo cual plantea la siguiente pregunta: ¿ese cambio de mirada que es la deconstrucción dimensional de las formas, y que se requiere en geometría y solamente en geometría, puede realizarse sin movilizar conocimientos geométricos?

Para comprender mejor esta pregunta, podemos volver a la columna de la izquierda en la tabla anterior (figura 3) y preguntarnos si es posible un análisis que no sea solamente visual y que no sea guiado por conocimientos geométricos. Vemos en seguida que el recurso de instrumentos para construir o para reproducir la forma rectangular ofrece un medio independiente de análisis.

En función de los instrumentos de los que se dispone para reproducir o para construir

Aquí el abanico de posibilidades no solamente es muy amplio, sino también muy heterogéneo.

Podemos distinguir por lo menos tres tipos (sin tener en cuenta los software de construcción) como lo indica la siguiente tabla. (Ver Figura 4).

Hay dos diferencias considerables entre los instrumentos que permiten manipulaciones materiales y los instrumentos que permiten un trazado gráfico.

Los primeros permiten voltear las piezas, ajustarlas dos a dos para ensamblarlas. Esas operaciones no son posibles con los instrumentos de trazado, principalmente los de tipo II.2. Pasar de los primeros a los segundos constituye un salto cognitivo considerable. En efecto, se pasa de un mundo en el que el espacio se organiza en función de los gestos del cuerpo y de su orientación, a un mundo en el que el espacio se determina en función de los gestos únicamente técnicos, es decir determinados por las posibilidades productivas de un instrumento.

Si sólo se producen trazos rectilíneos o curvilíneos (formas 1D), las herramientas de trazado permiten el ensamblaje por superposición de formas 2D. Pero surge entonces un problema que no existe cuando uno se contenta con manipular piezas de rompecabezas: el de la separación de las formas. Es verdad que se pueden superponer piezas apilándolas, pero esto no conduce a ninguna función visual 


\section{INSTRUMENTOS}

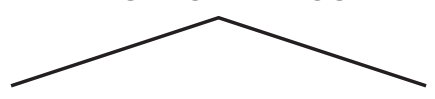

I. Que permiten manipulaciones de OBJETOS MATERIALES

I.1. Que producen formas 2D

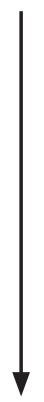

Piezas de

rompecabezas,

tangrams,

plegado de

papel, ... formas 1D

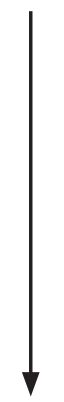

Pitillos
II. Que permiten operaciones

DE TRAZADO GRÁFICO

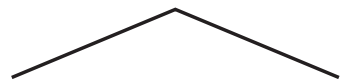

II.1. Que 9

producen I

formas 2D

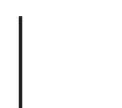

Sin Con

I.2. Que producen formas 1D producen
Instrumentos de manipulación libre pues su utilización en 3D responde a restricciones perceptivas y permite el paso de 2D a 1D
Instrumentos que exigen una manipulación "fina" pues su utilización depende de restricciones de instrucción que implican el paso de 0D a 1D

Figura 4. Clasificación de los instrumentos de construcción o de producción de formas.

de las piezas superpuestas, como en una figura producida gráficamente. Al cambiar de un tipo de instrumento a otro, ya no se juega el mismo juego. Con las operaciones de trazado con ayuda de instrumentos II.2 se entra en el modo de la representación gráfica que impone restricciones de reducción de representación, pero que también abre posibilidades cognitivas nuevas para la exploración geométrica.
La importancia de los instrumentos escogidos para las situaciones de reproducción en los primeros aprendizajes de la geometria

En general, en una actividad de reproducción no se le da importancia a la selección del instrumento. Sin embargo, las tareas de reproducción pueden ser radicalmente 
diferentes (tanto desde un punto de vista cognitivo como desde un punto de vista geométrico) según el tipo de instrumentos que se dan para reproducir una figura.
Reproducir un cuadrado con regla y compás o solamente con una parte de un molde de ese mismo cuadrado conduce a análisis completamente diferentes; las operaciones instrumentalmente posibles no serán en absoluto las mismas.

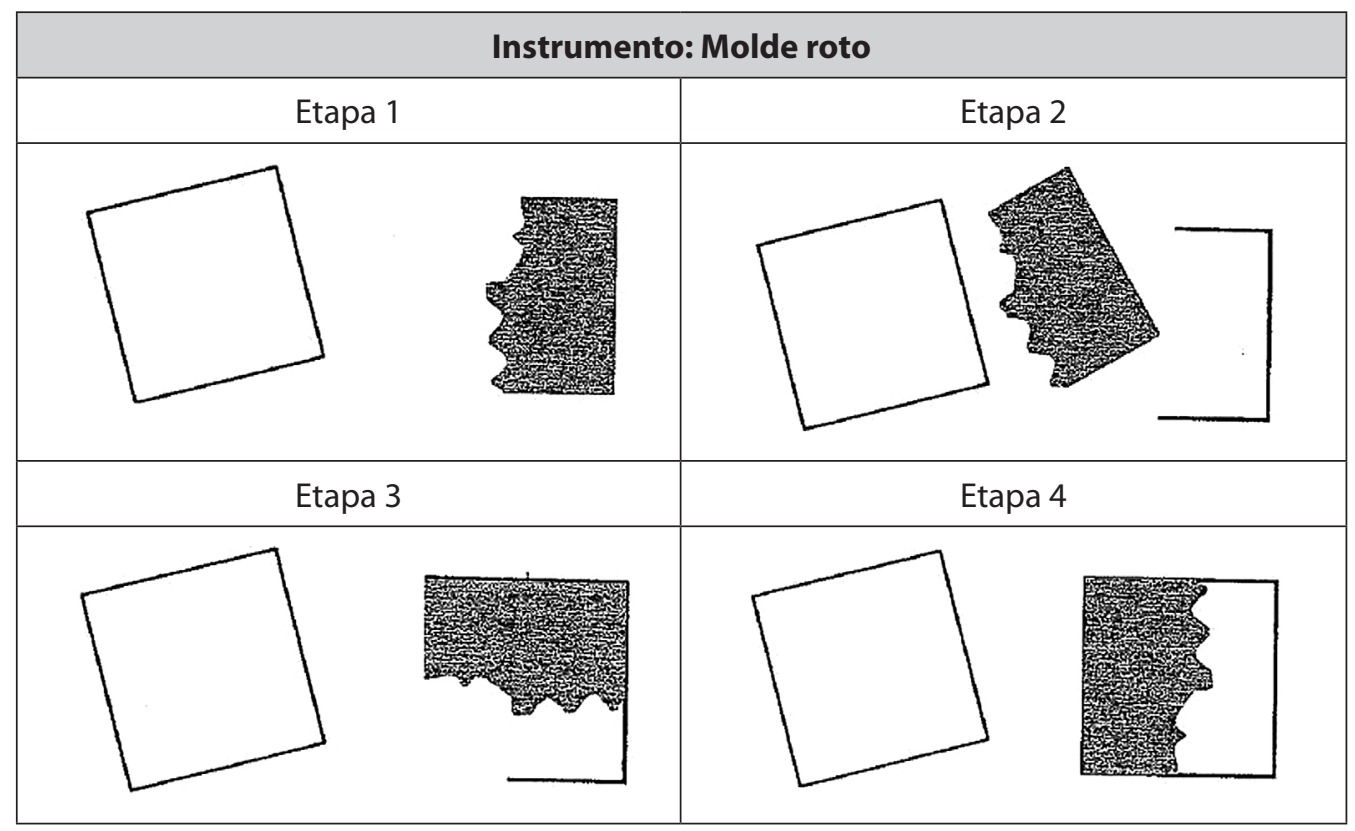

Figura 5. Reproducción de un cuadrado.

Este ejemplo muestra que lo importante no es la tarea de reproducción sino el tipo de instrumento escogido para la reproducción.

La diversidad de los instrumentos que pueden utilizarse en la enseñanza, de la cual podemos darnos una idea con la clasificación presentada anteriormente (figura cuatro), conduce a plantear cuatro preguntas principales.

- ¿Cuáles instrumentos utilizar y en qué orden utilizarlos en la organización de secuencias de actividades? Por ejemplo, la regla no debería ser el primer instrumento que se usa pues produce unidades visuales 1D.

- ¿La construcción de un tipo de figura debe estar asociado a la utilización de un instrumento específico o por el contrario es necesario poder construir una figura con instrumentos totalmente diferentes?

- ¿Para efectuar transferencias de longitud, hay que utilizar instrumentos que permitan medidas físicas? Esta pregunta se plantea porque la utilización de ins- 
trumentos que permiten medidas físicas conduce a neutralizar el aspecto visual de las figuras, focalizando la atención directamente sobre números y cálculos y por lo tanto a desviarse de las propiedades geométricas. Los procedimientos numéricos se sustituyen a los procedimientos geométricos.

- ¿Como los instrumentos conducen a aislar visualmente ya sea a formas 2D o formas 1D, cómo una selección de instrumentos puede hacer pasar a los alumnos de una visión perceptiva $2 \mathrm{D}$ a esta visión en 1D que se requiere para la identificación de propiedades geométricas? Es la selección del instrumento combinado con la selección de la figura la que crea la situación que permite a los alumnos hacer ese paso. Esta pregunta no es separable de otra: ¿"sobre qué tipo de figura hacer trabajar a los alumnos?"

Para que una secuencia de actividades de reproducción, organizada en función de una variación de instrumentos, pueda conducir a los alumnos a cambiar progresivamente su mirada, las figuras que se reproducen deben responder a cuatro criterios:

- Las figuras propuestas deben ser ensamblajes de formas y no solamente una "figura usual", es decir la forma típica de un polígono geométricamente notable (triángulo, cuadrado, rectángulo...). Estos ensamblajes de formas pueden hacerse por yuxtaposición o por superposición.

- Estos ensamblajes deben respetar alineamientos, pues el respeto de los alineamientos es importante para favorecer la actividad de prolongación de los trazados que es esencial para aprender a pasar de las superficies a las líneas en el análisis de las figuras.
Por ejemplo, los alumnos podrán pasar progresivamente de un análisis de las figuras como ensamblajes de superficies a un análisis como ensamblajes de líneas. Estos ensamblajes pueden también estar determinados por relaciones métricas.

- La selección de una figura, es decir de un ensamblaje que puede ser analizado como ensamblaje de superficies o como ensamblaje de líneas, no puede separarse del tipo de instrumento que se le asocia en la actividad que se propone a los alumnos: reproducirla, restablecerla...

- La posibilidad de un control por superposición de la igualdad de esas dos figuras es importante para darle sentido a toda actividad de reproducción de figura.

El trabajo sobre figuras que representan objetos geométricos simples (recta, cuadrado, triángulo) no permite que los alumnos entren en el juego de visualización matemática. Pero casi siempre, el trabajo para los alumnos entre 5 y 11 años se restringe a la identificación de tales figuras, lo que reduce el aprendizaje a la adquisición de un vocabulario.

Cómo modificar la mirada de los alumnos sobre una figura jugando con los instrumentos que se utilizan

Los instrumentos que se emplean para reproducir una figura dada dirigen la manera de mirar.

Vemos que algunos instrumentos (moldes, plantillas) conservan la prioridad perceptiva 2D, mientras que otros sólo pueden utilizarse si uno es capaz de sustituir a esta percepción dominante la visualización de una red de formas 1D (regla, compás). Las 
Los cambios de mirada necesarios sobre las figuras Raymond Duval | Traducción: Martín Eduardo Acosta Gempeler

tareas de reproducción van a ser radicalmente diferentes si se utilizan instrumentos que producen formas $1 \mathrm{D}$ o formas 2D. Por eso surge una pregunta más práctica para la organización de las actividades: ¿cómo, a partir de instrumentos que permiten reproducir formas 2D, preparar a los alumnos a la deconstrucción dimensional de las formas 2D, sin la cual la utilización de instrumentos que producen formas 1D corre el riesgo de permanecer ciega?

Para poner en evidencia el rol de esta variable didáctica que es la variación de los instrumentos, vamos a tomar como ejemplo una figura simple -un triángulo- y no una figura que aparece como un ensamblaje de formas como lo exigiría el primer criterio indicado anteriormente. Volveremos más adelante a la otra variable, la selección de las figuras.

Ejemplos de reproducción de un triángulo sin los instrumentos convencionales de geometría

Construir o reproducir una figura simple significa aquí trazar el contorno cerrado de la forma simple; el instrumento sirve para guiar el lápiz. Para evaluar la calidad de la reproducción, se recurre al principio de superposición del modelo y de su realización. Vamos a presentar ejemplos muy diferentes de reproducción de un triángulo, utilizando por lo menos un instrumento productor de formas 2D: plantillas rotas. Esas herramientas de dos dimensiones pueden a priori utilizarse simultáneamente o no, pero la utilización, de una sola herramienta a la vez, obliga a una deconstrucción 1D de la figura a reproducir (lo que se ve en el ejemplo tres, comparándola con el ejemplo dos).

Los cinco ejemplos siguientes varían en tres aspectos:
- Los instrumentos utilizados son o no independientes de la figura a reproducir.

- El número de herramientas disponibles en un momento dado (molde o plantilla).

- El uso de un instrumento 2D con un solo borde rectilíneo que puede contener una información o no. La información consiste en trazos rectilíneos que se hacen sobre el instrumento, para permitir transferencias de longitud o transferencias de ángulo y de dirección. Cuando este instrumento 2D no tiene ningún trazo lo llamaremos regla no informativa; cuando hemos colocado uno o varios trazos, diremos que se informó la regla y hablaremos de regla informativa.

\section{Ejemplo uno}

Se trata de reproducir un triángulo utilizando como plantilla el molde roto del triángulo. (Ver Figura 6).

El gesto gráfico se inscribe aquí en la concepción del contorno cerrado de una figura 2D característica del molde o plantilla. Dar un molde roto obliga a interrumpir la continuidad de ese gesto gráfico (etapa dos). Para concretarlo hay que recurrir a la plantilla (etapa tres).

\section{Ejemplo dos}

Para reproducir un triángulo, se rompe un molde y se entregan varias reglas. (Ver Figura 7).

Al igual que en el ejemplo anterior, la utilización del molde roto conduce a un trazado incompleto, pero aquí para completarlo hay que utilizar dos reglas no informativas en lugar de una plantilla. La necesaria superposición de estas dos reglas para cons- 
118

T $\in \Delta$ Tecné, Episteme y Didaxis

No. 27 • Primer semestre de 2010 • pp. 108-129

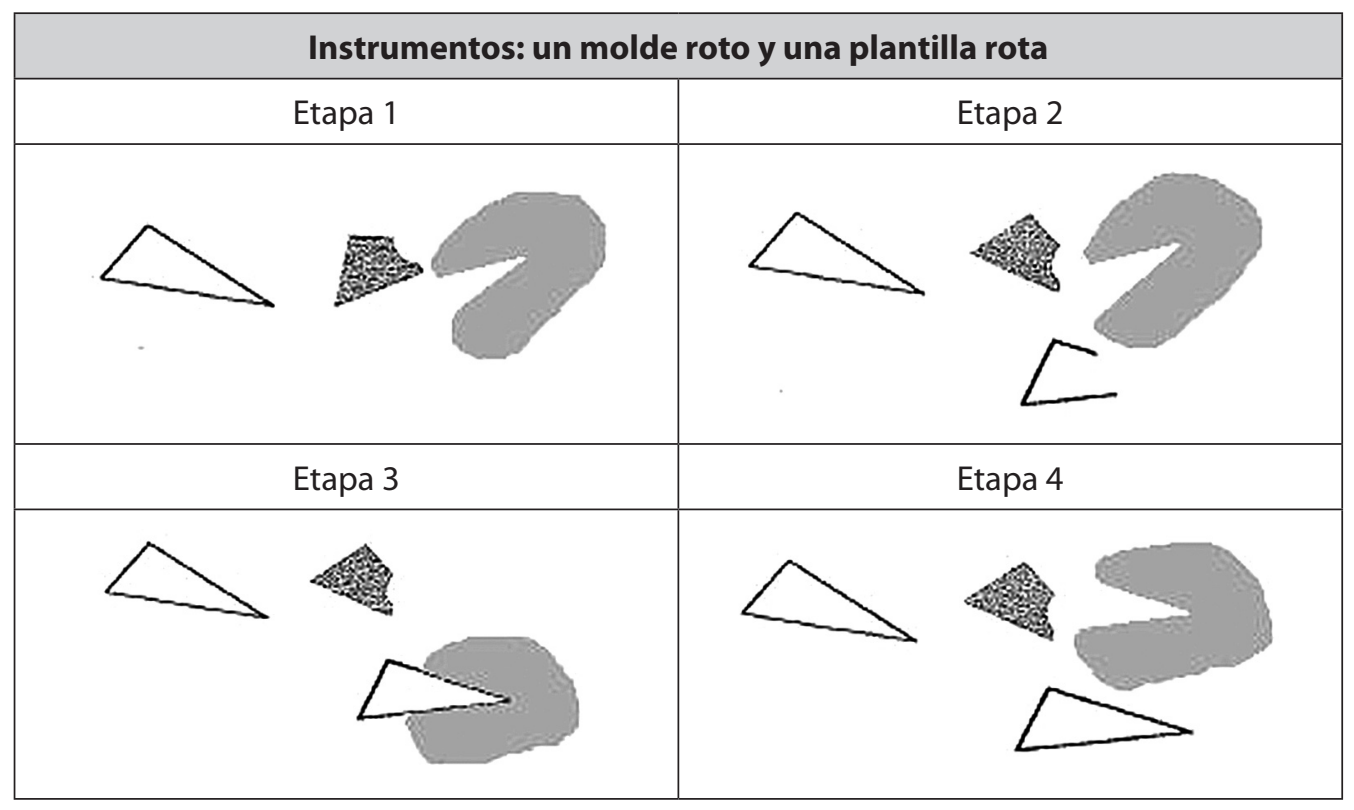

Figura 6.

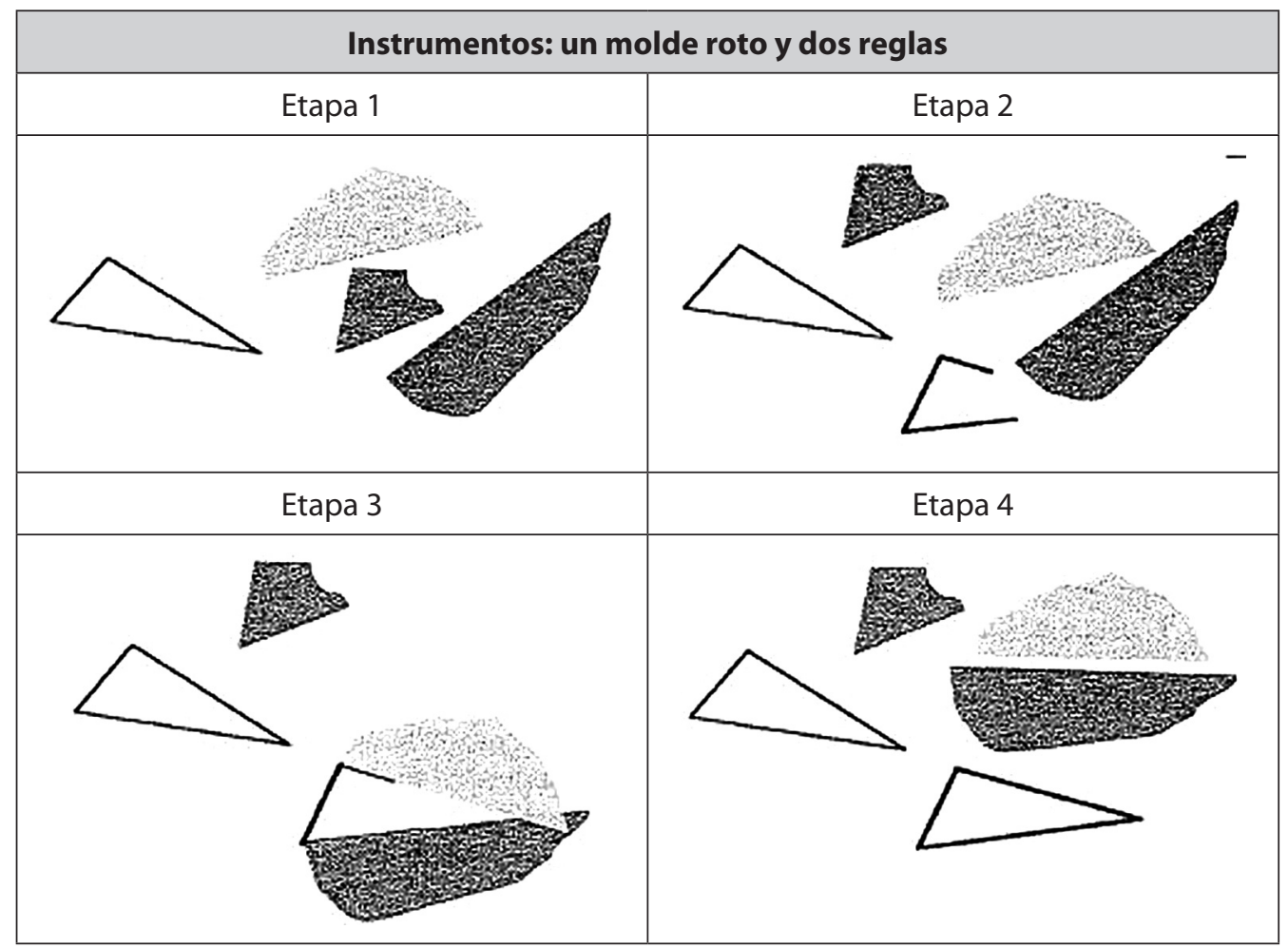

Figura 7. 
Los cambios de mirada necesarios sobre las figuras Raymond Duval | Traducción: Martín Eduardo Acosta Gempeler

truir la plantilla prepara la capacidad de prolongar una línea. (Ver etapa tres del ejemplo tres, etapa dos del ejemplo cuatro y etapa dos del ejemplo cinco).

\section{Ejemplo tres}

En el ejemplo anterior, la supresión de una regla obliga a abandonar la estrategia de reconstrucción de la plantilla, y abandonar también el grafismo del contorno externo del triángulo, para trazar sucesivamente los dos lados que faltan. EI hecho de que sólo haya una regla obliga igualmente a trazar no solamente un lado sino a trazar también su prolongación. El segmento trazado en la etapa cuatro es el primer elemento $1 D$.

En los ejemplos anteriores, los elementos trazados sólo eran una parte del contorno cerrado (forma 2D). En esta situación, desde un punto de vista gráfico, el contorno del triángulo explota en partes de ese contorno. Hay una primera etapa en el paso complejo de 2D a 1D.

\begin{tabular}{|c|c|c|}
\hline \multicolumn{3}{|c|}{ Instrumentos: un molde roto y una sola regla } \\
\hline Etapa 1 & Etapa 2 \\
\hline & & \\
\hline
\end{tabular}

Figura 8. 


\section{Ejemplo cuatro}

¿Cómo hacer cuando la forma de los instrumentos no tiene ninguna relación con el contorno de la figura que se debe reproducir?

El trazado de líneas sobre la superficie cualquiera en la etapa tres de la siguiente tabla puede aparecer como una operación sustitutiva de las acciones de cortado o de doblado necesarias para obtener un molde del triángulo o, en su defecto, un molde roto del triángulo.

La capacidad de superponer una superficie cualquiera sobre la figura por reproducir, y la de inscribir un pedazo de cada lado de esta figura, de manera que se pueda reproducirla de una sola vez por la prolongación de esos pedazos, son indicadores de una visualización geométrica avanzada.

\begin{tabular}{|c|c|}
\hline \multicolumn{2}{|c|}{ Instrumentos: una superficie cualquiera y una regla no informativa } \\
\hline Etapa 1 & Etapa 2 \\
\hline Etapa 3 & \\
\hline Etapa 5 & Etapa 4 \\
\hline
\end{tabular}




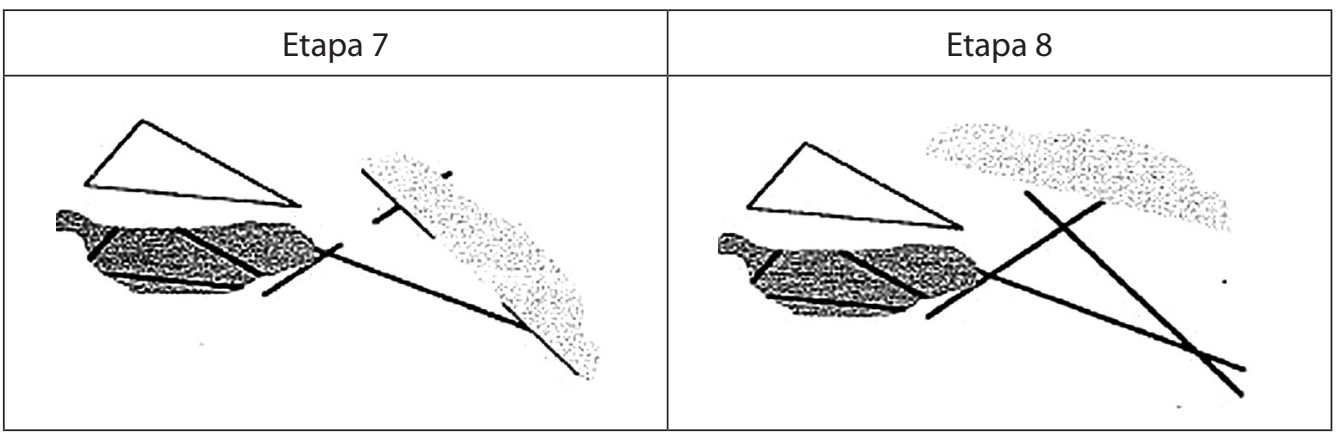

Figura 9.

Ejemplo cinco

¿En ausencia de un molde y de una plantilla del triángulo, cómo reproducir si se dispone de tres reglas no informativas que pueden volverse informativas?

Sabiendo que una plantilla podría bastar...

\begin{tabular}{|c|c|}
\hline \multicolumn{2}{|c|}{ HERRAMIENTAS: 3 reglas no informativas } \\
\hline Etapa 1 & Etapa 2 \\
\hline & \\
\hline
\end{tabular}

Figura 10. 
La tarea de reproducción implica tres tipos de acciones:

- Superponer las reglas de manera que se reconstruya una plantilla (etapa dos).

- Trazar tres líneas sobre dos de las reglas (etapa tres).

- Deslizar una de las tres reglas sobre uno de los trazos (etapa cuatro).

Vemos entonces que en esta tarea las reglas se colocan por yuxtaposición al triángulo, y están relacionadas entre ellas por superposición. Los tres trazos hechos sobre las reglas conservan la memoria de las superposiciones.

Esta manera de utilizar reglas introduce automáticamente la operación de prolongación de un trazado, aunque en un primer momento esto no nos saca del marco de la figura a reproducir. Pero lo importante es que así se rompe la unidad de contorno y la unidad visual de cada uno de los tres bordes.

En este ejemplo de procedimiento hay dos reglas informadas. El paso de la etapa cuatro a la etapa cinco muestra que habríamos podido hacer informativa una sola regla, y las otras dos habrían servido para reconstituir el molde.

Reconocemos aquí uno de los casos de igualdad de los triángulos que se encuentra en Euclides. (Libro uno, proposición 26).

\section{Ejemplo de reproducción de un triángulo con instrumentos convencionales de geometría}

\section{Ejemplo seis}

La escuadra, herramienta convencional que permite trazar elementos 1D, es un molde de triángulo rectángulo, pero no se utiliza para desplazar una forma 2D. En efecto, sólo los dos lados rectilíneos se tienen en cuenta y no se lo utiliza como una regla informativa. No se hacen rayas sobre una escuadra.

Sin embargo, puede ser utilizada como una parte de un molde de cuadrado o de rectángulo. (Ver Figura 11).

En este ejemplo, la escuadra es un instrumento para trazar líneas, es decir elementos 1D, que son perpendiculares. Aquí, la actividad de reproducción es más compleja pues hay que hacer aparecer un trazo suplementario sobre la figura simple del triángulo a reproducir. En otras palabras, hay que transformar la figura simple inicial en una figura ensamblada. El triángulo debe verse como una yuxtaposición (ver figura 1) de dos triángulos rectángulos, triángulos formalmente desplazables y reproducibles gracias a la escuadra.

A menudo las figuras dadas para reproducir no permiten que los alumnos aborden el paso de 2D a 1D pues contienen una esquina recta o dos segmentos perpendiculares. En el caso de una figura con una esquina recta, la escuadra se utiliza como un molde, es decir como una herramienta 2D.

En el caso contrario, la escuadra debe utilizarse como una herramienta 1D que permite trazar sobre la figura modelo un trazo recto 1D perpendicular a otro. (Ver etapa 2 de la figura 11).

Esta ambigüedad de la escuadra conduce a preguntarnos sobre la utilización efectiva de los otros instrumentos por parte de los alumnos. La utilización de los instrumentos de reproducción, como los moldes, se hace por superposición y por lo tanto por aproximación perceptiva, y podemos preguntar- 
Los cambios de mirada necesarios sobre las figuras Raymond Duval | Traducción: Martín Eduardo Acosta Gempeler

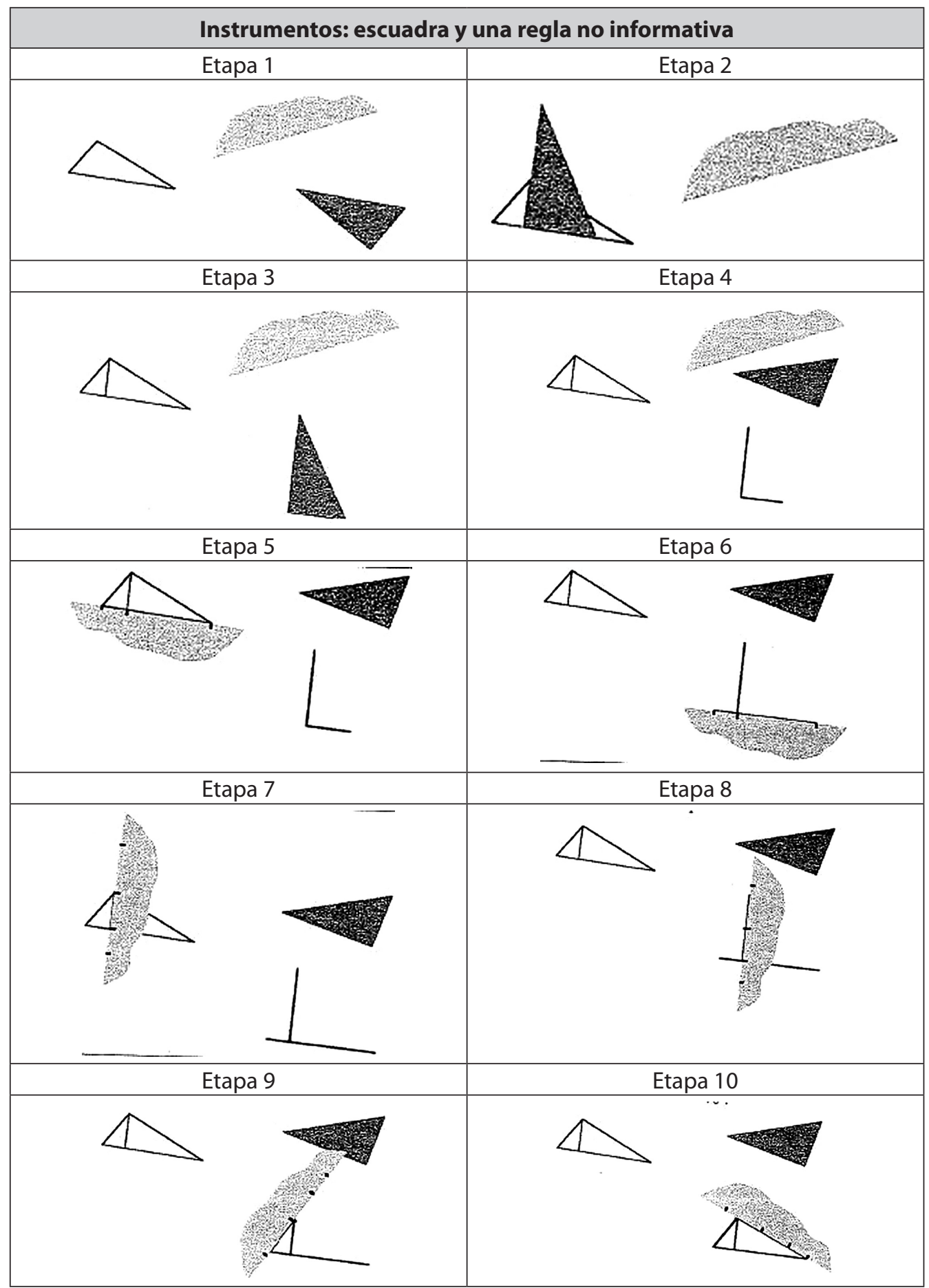

Figura 11. 
nos si los alumnos, que no pueden efectuar o que no han tomado conciencia del cambio de mirada implicada por el paso de las formas 2D a las formas 1D, no buscan utilizar los instrumentos de construcción como si se tratara de moldes.

Es una de las constataciones mayores que surge del estudio de Bouleau (2001:29): "a partir de un cierto umbral de competencia de trazado... los alumnos no logran unir los puntos con la regla y vuelven al dibujo a mano alzada".

\section{Selección de los instrumentos y objetivos de aprendizaje}

Los alumnos del ciclo uno (pre-escolar a segundo) han tenido ocasión de trazar figuras a partir de un molde o de una plantilla. En el ciclo dos (tercero a quinto), deben aprender a trazar figuras utilizando los instrumentos convencionales. Tal utilización sólo puede hacerlos sensibles a las restricciones geométricas internas de las figuras reproducidas o construidas si pueden desconstruir visualmente las formas $2 \mathrm{D}$ en elementos 1D.

Esta deconstrucción dimensional, que es cognitivamente muy compleja, no parece poder adquirirse únicamente por la utilización de instrumentos que producen elementos 1D. Es la utilización de instrumentos diferentes, unos que permiten transportar informaciones 2D, otros solamente informaciones 1D, la que va a permitir a los alumnos entrar progresivamente en la deconstrucción dimensional de las formas 2D, que es una condición para la explicitación de los conocimientos geométricos. En efecto, los conocimientos geométricos presuponen una articulación entre la visualización y el lenguaje, ya sea que éste sea utilizado para descripción, distinción (subyacentes a todo vocabulario) o justificación, que sólo se encuentra en geometría y en ningún otro campo de conocimiento (Duval, 2005).

La organización de actividades para hacer entrar los alumnos en este proceso cognitivo fundamental para los saberes geométricos implica entonces una progresión sobre los instrumentos que se utilizan para reproducir figuras, como pudimos ver en la secuencia de los ejemplos precedentes:

- Moldes y plantillas.

- Moldes y varias reglas (superficie con un solo borde rectilíneo informarle)

- Molde y una sola regla.

- Superficie cualquiera y una regla.

- Únicamente reglas.

- Una regla y una escuadra

En la secuencia de ejemplos precedente, la progresión presentada se hizo únicamente en función de la variable "instrumentos". Para poder organizar en clase actividades que desarrollen un cambio de mirada, hay que combinarla con la selección apropiada de las figuras a reproducir y también determinar las condiciones en las que se pide una tarea de reproducción.

\section{Referencias para la organización de actividades}

La selección de los instrumentos debe replantearse en el contexto de las dos preguntas que siguen:

- ¿Qué tipos de figura escoger?

- ¿Cómo motivar a los alumnos para que se apropien de la tarea? 
Los cambios de mirada necesarios sobre las figuras Raymond Duval | Traducción: Martín Eduardo Acosta Gempeler

\section{Qué tipos de figura escoger}

Vamos a utilizar dos criterios.

Las figuras seleccionadas deben poder ser vistas como un ensamblaje de formas por yuxtaposición y como un ensamblaje por superposición.

Un polígono cóncavo o una figura ensamblaje de partes cóncavas y convexas parecen ser excelentes figuras candidato. El hecho de ser una figura cerrada es en efecto uno de los principales factores de organización de elementos del campo perceptivo en una unidad figural (Kanizsa, 1998:40-41). Es lo que conduce a ver espontáneamente toda forma cóncava en su entorno convexo, y por lo tanto a completarla.

Las figuras elegidas deben obligar a prolongar líneas o a construir nuevas líneas para lograr la reproducción.

La prolongación de líneas prepara los alumnos a investigaciones posteriores sobre alineación.

Aquí hay un ejemplo de figura que responde a esos dos criterios. Se notará en particular que la figura escogida centra la actividad en las prolongaciones de líneas y en las relaciones de longitudes. (Ver Figura 12).

La figura a reproducir fue seleccionada para que el polígono cóncavo pueda descomponerse en dos triángulos (etapas 2:03). Nótese sobre todo la importancia de la etapa seis: la colocación de la regla opaca sobre la figura modelo constituye un enriquecimiento de esta figura. En efecto, es lo mismo que descomponer el cuadrilátero en dos partes: una parte oculta (un triángulo) y una parte visible (un triángulo y un nuevo cuadrilátero). Esta división impone una prolongación. Toda esta operación aplica una deconstrucción dimensional. Va a permitir luego una transferencia de medida (etapa siete) sobre un segmento que, en el modelo a reproducir, no estaba ni totalmente trazado ni era elemento del contorno.

Para tomar en cuenta el segundo criterio que toca directamente la descomposición dimensional de las formas, es muy eficaz una actividad de restauración de figuras, y no solamente de reproducción. Además del modelo, se da una reproducción ya comenzada, es decir una versión del modelo en la que se han borrado algunos trazos, de tal forma que el alumno sólo tenga que completar. Pero comenzando así la reproducción, se imponen ciertos algoritmos de reproducción. Vemos entonces que la actividad de restauración es una variable didáctica suplementaria según el número y las propiedades de los trazos que se borraron (Perrin, 2004).

\section{Cómo motivar a los alumnos para que se apropien de las tareas de reproducción o de restauración el juego de los malos}

Las actividades de reproducción o de restauración de figuras representan tareas diferentes según los instrumentos utilizados. Hemos mostrado cómo una progresión en el uso de los instrumentos en relación con las figuras visualmente ricas hace entrar en el juego de la deconstrucción dimensional de las formas. Para que esto sea verdaderamente operacional, es necesario que los alumnos se vean obligados a tomar conciencia de las posibilidades y las restricciones de cada instrumento utilizado, es decir al hecho de que la utilización de instrumentos diferentes conduce a procedimientos diferentes. Es entonces interesante hacer diferenciar el costo de cada procedimiento 
T $\in \Delta$ Tecné, Episteme y Didaxis

No. 27 • Primer semestre de 2010 • pp. 108-129

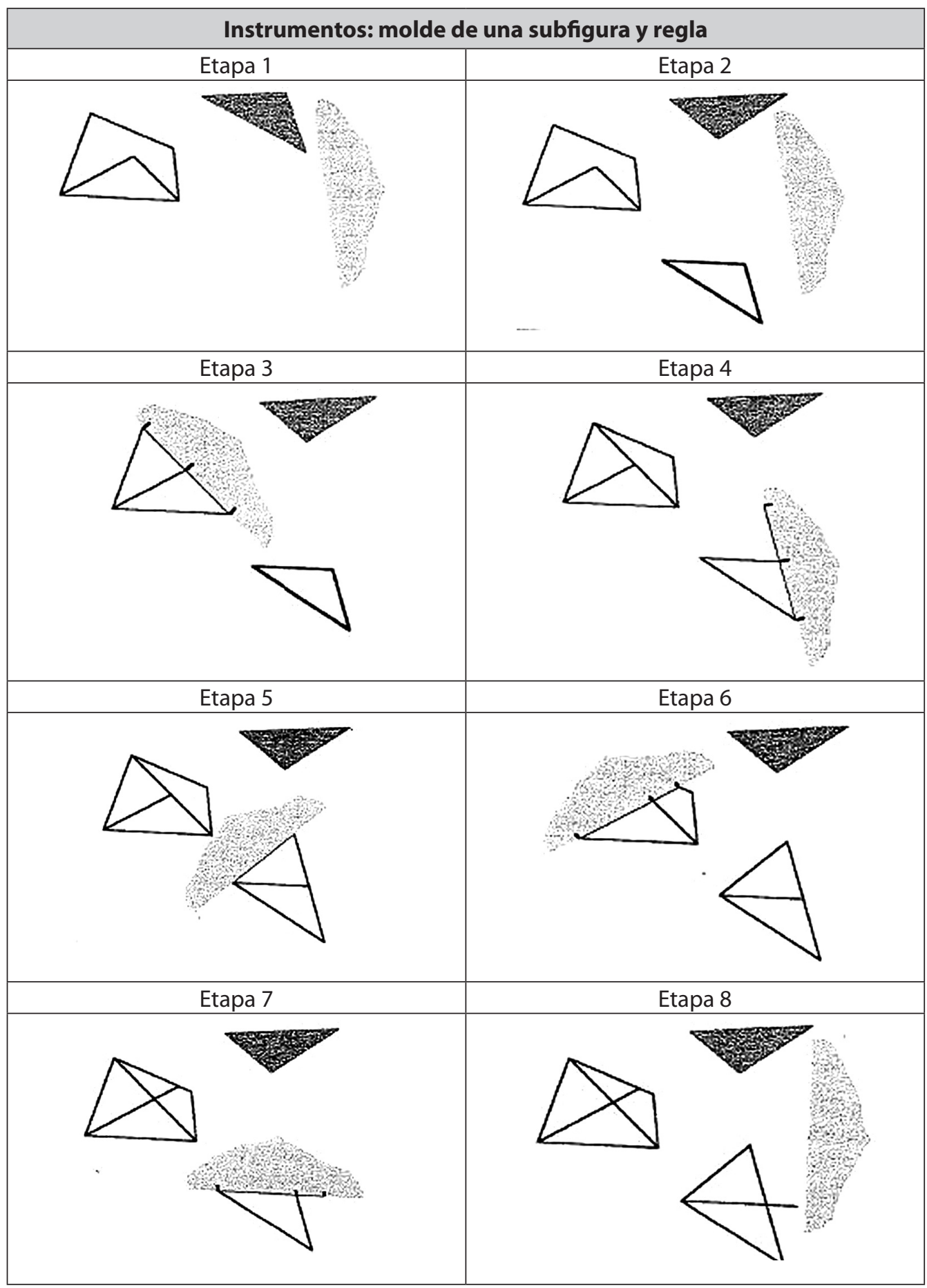




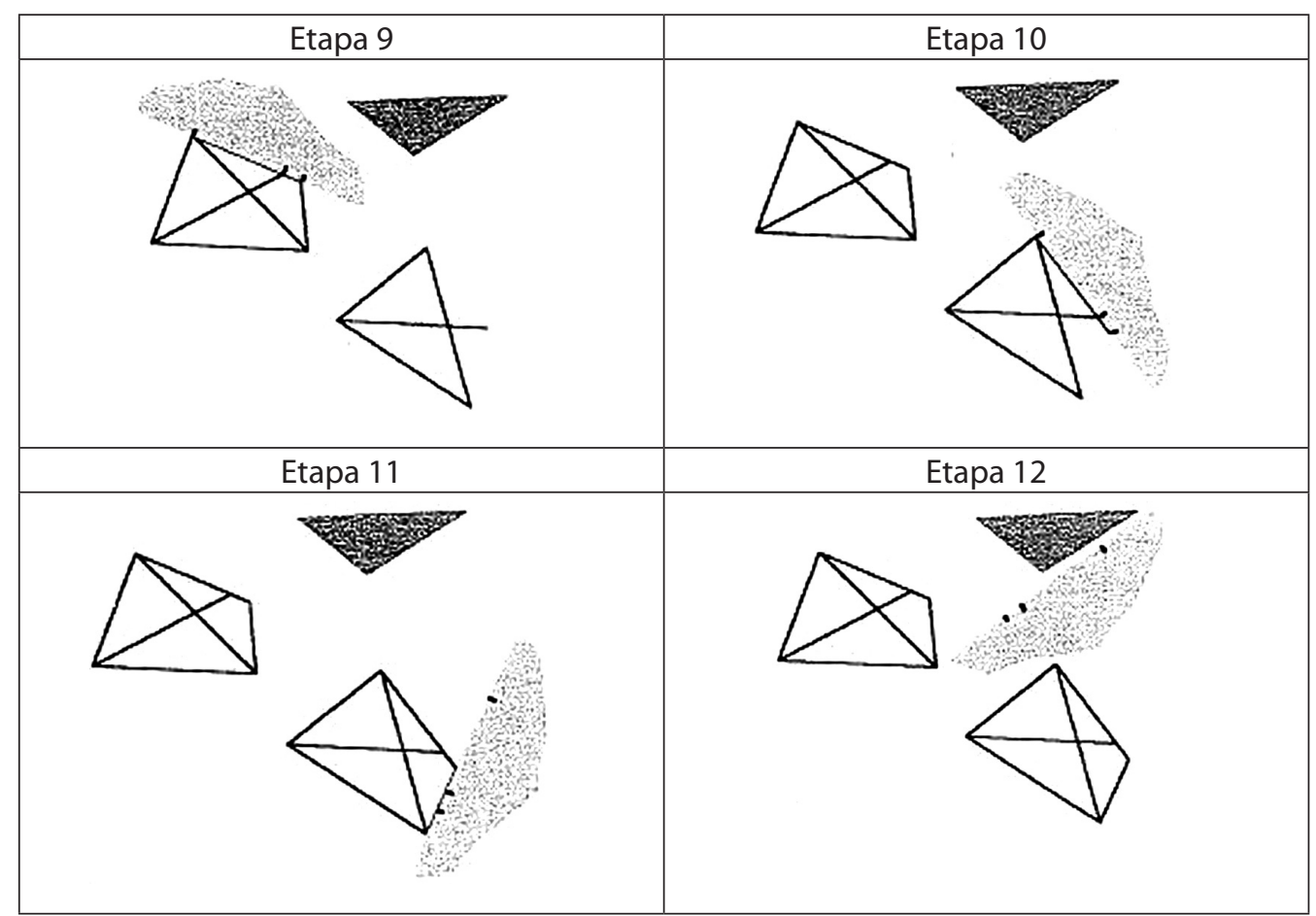

Figura 12.

por un juego de puntos. Para una tarea dada, de reproducción o de restauración, los alumnos pueden disponer de varios instrumentos y pueden también pedir ayuda (por ejemplo información sobre la posición de un instrumento). Cada utilización de un instrumento, y cada solicitud de ayuda, cuesta un cierto número de puntos. No se trata solamente de reproducir o de restaurar, sino de hacerlo con el menor costo posible.

Un grupo de Profesoras de CE2, después de un curso de formación, trató de poner sistemáticamente en obra a actividades de análisis de figuras. Hicieron trabajar a los alumnos sobre figuras que habían elaborado después de la formación y utilizaron el juego de puntos. A continuación hay un extracto de una entrevista muy larga sobre su experiencia, que muestra cómo ellas pudieron percibir la evolución de sus alumnos. "lo que nos pareció verdaderamente muy, muy interesante, muy motivante para los alumnos, era ese sistema de puntos buenos y malos... que la meta del juego era tratar de tener la menor cantidad de puntos posible, construir su figura con la menor cantidad de puntos posible... en las primeras sesiones, empieza inmediatamente la reproducción... después, al cabo de algunas sesiones, comprenden verdaderamente el interés del análisis y después se toman un buen tiempo de análisis, y no comienza la reproducción antes de haber visto puntos relacionados, puntos medios... les gusta mucho... si si les gusta mucho buscar puntos, es un poco como pistas, deben buscar, deben encontrar cosas, saben que van a encontrar cosas, y por lo tanto se enfrentan a una situación problema... si es lúdico, es... es una investigación y por lo tanto están en acti- 
vidad, son muy activos incluso los niños en dificultad encuentran cosas... tienen verdaderamente ganas de lograrlo y de lograrlo bien. $Y$ están en competencia no necesariamente con los otros, lo sentimos verdaderamente en competición con ellos mismos. Pienso que es también el gusto que se les dio. Es verdad que esas actividades dan mucho gusto..."

\section{Conclusión}

La introducción de conocimientos geométricos implica una deconstrucción dimensional que es imposible sin un cambio en la manera habitual de mirar las figuras. Uno de los objetivos de las primeras actividades geométricas es introducir a los alumnos en este cambio que no pueden sospechar. Hemos visto que la organización de actividades para alcanzar este objetivo combina una selección de figuras apropiadas y una variación en los instrumentos utilizados para reproducirlas.

Lo que llamamos las figuras-ensamblajes tiene un rol clave para hacer descubrir procesos de deconstrucción de formas 2D, que permiten también enriquecer una figura. En efecto, una figura-ensamblaje posibilita una descomposición visual en dos tiempos:

- Descomponer en formas 2D que permiten obtener esa figura por yuxtaposición o por superposición

- Reconocer la presencia de un elemento del borde, común a dos contornos cerrados, lo que equivale a aislar dos formas en las que es borde.

La utilización de instrumentos clásicos (regla, escuadra) presupone esta deconstrucción, que es opuesta a la manera de ver de los alumnos. En efecto, las actividades con regla y escuadra (utilizadas de manera convencional) sólo son posibles si una figura 2D puede descomponerse en elementos 1D. Esta descomposición exige que los alumnos hayan notado no solamente las relaciones topológicas entre esos elementos 1D sino también sus relaciones de alineación y sus relaciones métricas.

Para ayudar a los alumnos a tomar conciencia de ese juego de visualización geométrica que es el paso de 2D a 1D, hay que utilizar instrumentos que permitan descomponer una figura-ensamblaje en otras formas $2 D$ que se yuxtaponen o se superponen. Todo el mundo piensa evidentemente en las plantillas. Pero para que su uso sea didácticamente eficaz, hay que proponer plantillas incompletas. La tarea de reproducción cambiará según la importancia de lo que falta. Pero esa es una variable a disposición del profesor para construir una progresión.

En los diferentes ejemplos presentados, las figuras eran cualesquiera, pero obligaban a hacer un análisis en términos de buscar las prolongaciones o alineamientos. Puede ampliarse el campo proponiendo figuras simétricas o proporcionales. En ese caso, las tareas de reproducción, de restauración o de construcción se convierten en actividades para hacer surgir también propiedades geométricas, pero esa será la siguiente etapa.

\section{Referencias bibliográficas}

Bouleau, N. (2001). Reproduction de figures et géométrie en cycle 1 et 2 . Grand N. No. 67. 15-32.

Duval, R. (2005). Les conditions cognitives de l'apprentissage de la géométrie: développement de la visualisation, différenciations des raisonnements et coordination 
de leurs fonctionnements. Annales de didactique des mathématiques et de sciences cognitives, 10, 5-55.

Godin, M. (2004). De trois regards posibles sur une figures au regard "géométrique", Actes du séminaire national de didactique des mathématiques. ADIREM et IREM de Paris 7, p. 39-70.

Euclide. (1990). Les Elements, V. I (tr. B. Vitrac). Paris: P.U.F.

Kanizsa, G. (1998). La grammaire du voir (tr. A. Chambolle). Paris: Diderot éditeur.
Peirce, Ch. S., (1978), Ecrits sur le signe. Paris: Le Seuil.

Perrin-Glorian, M. J. (2004). Des situations pour apprendre à regarder les figures. Quelques resultants et perspectives. Actes du séminaire national de didactique des mathématiques. ADIREM et IREM de Paris 7, p. 71-89.

Verbaere, O.; Gaudeul, C. (2005). Entretien sur un stage en géométrie, Document IUFM centre de Lille. 